\title{
ČECH-COMPLETENESS AND RELATED PROPERTIES OF THE GENERALIZED COMPACT-OPEN TOPOLOGY
}

\author{
LUBICA HOLÁ AND LÁSZLÓ ZSILINSZKY
}

\begin{abstract}
The generalized compact-open topology $\tau_{C}$ on partial continuous functions with closed domains in $X$ and values in $Y$ is studied. If $Y$ is a noncountably compact Čech-complete space with a $G_{\delta}$-diagonal, then $\tau_{C}$ is Čechcomplete, sieve complete and satisfies the $p$-space property of Arhangel'skiř, respectively, if and only if $X$ is Lindelöf and locally compact. Lindelöfness, paracompactness and normality of $\tau_{C}$ is also investigated. New results are obtained on Čech-completeness, sieve completeness and the $p$-space property for the compact-open topology on the space of continuous functions with a general range $Y$.
\end{abstract}

\section{INTRODUCTION}

The generalized compact-open topology $\tau_{C}$ on the space of partial continuous functions with closed domains was introduced by $J$. Back in [Ba] in connection with investigating utility functions emerging in mathematical economics. It proved to be a useful tool in studying convergence of dynamic programming models [Wh], $[\mathrm{La}]$, as well as in applications to the theory of differential equations [BC]. This new interest in $\tau_{C}$ complements the attention paid to spaces of partial maps in the past [Za], [Ku1], [Ku2], [AA], [AB], [BB], [Se], and more recently in [Fi], [St], [KS], [BCH1], [BCH2], [DN1], [DN2], [HZ1], [HZ2].

Various topological properties of $\tau_{C}$ have already been established, e.g. separation axioms in [Ho1], complete metrizability in [Ho2] and other completeness type properties in [HZ1] and [NZ], respectively; also, in [DN1], [DN2] the authors study topological properties of spaces of partial maps in a more general setting.

It is the purpose of this paper to continue in this research by investigating Cechcompleteness of $\tau_{C}$. In the process, sieve completeness and the $p$-space property [Arh] of $\tau_{C}$ is proved to be equivalent to its Čech-completeness in the most interesting cases, which is much like the situation with the compact-open topology $\tau_{C O}$ on the space of continuous functions (see [MN1, MN2]). It is worth noticing however, that despite the close connection between $\tau_{C}$ and $\tau_{C O}$, properties of these topologies do not always coincide. On the contrary, in some cases (like Baireness, or weak $\alpha$-favorability [HZ1]), the generalized compact-open topology exhibits properties resembling those of the Fell hyperspace topology $\tau_{F}$, which makes $\tau_{C}$ a true mixture of $\tau_{C O}$ and $\tau_{F}$. In Section 2 we collect some definitions and auxiliary results for the topologies we are to study. In Section 3, we prove the main results of the

2000 Mathematics Subject Classification. Primary 54C35; Secondary 54E18, 54E50, 54D20, 54B20, 54C60, 54D99.

Key words and phrases. Generalized compact-open topology, Fell topology, compact-open topology, Čech-complete, sieve complete, $p$-spaces, Lindelöf, paracompact and normal spaces.

This work has been supported by the grant Vega 2/7139/27. 
paper: a characterization of Čech-completeness, sieve completeness and the $p$-space property, respectively. Extending a theorem of Holá [Ho2], a full characterization of complete metrizability of $\tau_{C}$ is also given. In Section 4, we obtain sufficient conditions for Čech-completeness, sieve completeness and the $p$-space property, respectively, of the compact-open topology $\left(C(X, Y), \tau_{C O}\right)$ for $Y$ with a $G_{\delta}$-diagonal, thus generalizing results of McCoy and Ntantu [MN1, MN2]. In Section 5, the relationship between Lindelöfness, paracompactness and normality of $\tau_{C}$ is explored. Surprisingly, normality of $\tau_{C}$ implies its Cech-completeness and the reverse implication is also true under some restrictions. An application of paracompactness of $\tau_{C}$ to continuous extensions of partial functions is given.

\section{Preliminaries}

Unless otherwise noted, all spaces are nontrivial Hausdorff spaces. If $X$ is a topological space, then $B^{c}$, $\operatorname{int} B$, and $\bar{B}$ (or $\bar{B}^{X}$ ) will stand for the complement, interior, and closure of $B \subseteq X$, respectively. Denote by $C L(X)$ the family of nonempty closed subsets of $X$, and by $K(X)$ the nonempty compact subsets of $X$. For any $B \in C L(X)$, and a topological space $Y, C(B, Y)$ will stand for the space of continuous functions from $B$ to $Y$ (so-called partial maps). Denote by

$$
\mathcal{P}=\mathcal{P}(X, Y)=\bigcup\{C(B, Y): B \in C L(X)\}
$$

the family of all partial maps. We will identify a partial map $f$ with its graph $\Gamma(f) \in C L(X \times Y)$. If $Y$ is a Tychonoff space and $c Y$ is a fixed compactification of $Y$, then $\mathcal{P} \subseteq C L(X \times c Y)$, since if $\left(x_{\lambda}, f\left(x_{\lambda}\right)\right)$ is a net in $\Gamma(f) \in \mathcal{P}$ converging to some $(x, y) \in X \times c Y$, then $x_{\lambda} \rightarrow x$ and $f\left(x_{\lambda}\right) \rightarrow y$; further, by continuity of $f$, $f\left(x_{\lambda}\right) \rightarrow f(x)$, so $y=f(x)$ and $(x, y) \in \Gamma(f)$.

Define the so-called generalized compact-open topology $\tau_{C}$ on $\mathcal{P}$ as the topology having subbase elements of the form

$$
\begin{aligned}
{[U] } & =\{f \in \mathcal{P}:(\operatorname{dom} f) \cap U \neq \emptyset\}, \\
{[K: I] } & =\{f \in \mathcal{P}: f(K \cap(\operatorname{dom} f)) \subseteq I\},
\end{aligned}
$$

where $U$ is open in $X, K \in K(X)$ and $I$ is an open (possibly empty) subset of $Y$. We can assume that the $I$ 's are members of some fixed open base for $Y$, or empty.

The compact-open topology [En, MN1] $\tau_{C O}$ on $C(X, Y)$ has subbase elements of the form $\{f \in C(X, Y): f(K) \subseteq I\}$, where $K \in K(X)$ and $I \subseteq Y$ is open.

Denote by $\tau_{F}$ the so-called Fell topology [Be, KT] on $C L(X)$ having subbase elements of the form

$$
V^{-}=\{A \in C L(X): A \cap V \neq \emptyset\}
$$

with $V$ open in $X$, plus sets of the form

$$
\left(K^{c}\right)^{+}=\left\{A \in C L(X): A \subseteq K^{c}\right\},
$$

with $K \in K(X)$. It was observed by Fell that if $X$ is locally compact, then $\left(C L(X) \cup\{\emptyset\}, \tau_{F}\right)$ is a compact Hausdorff space [Fe]; furthermore, since $C L(X)=$ $X^{-}$is an open subspace of $\left(C L(X) \cup\{\emptyset\}, \tau_{F}\right),\left(C L(X), \tau_{F}\right)$ is locally compact, if $X$ is. We will use $\tau_{F}$ to denote the Fell topology on $C L(X \times c Y)$ as well as on $\mathcal{P}$. If $L \in C L(X \times Y)$, denote

$$
L[x]=\{y \in Y:(x, y) \in L\}
$$


(i.e. $L$ can be viewed as a multifunction with a closed graph [HM]). Let $p_{X}$ be the projection map from $X \times Y$ onto $X$, and $\omega$ the non-negative integers. For notions not defined in the paper see [En].

In the following two propositions we explore the relationship between the various topologies defined in this section:

\section{Proposition 2.1.}

(i) $X$ and $\left(C L(X), \tau_{F}\right)$ embed in $\left(\mathcal{P}, \tau_{C}\right)$; further, they embed as closed subsets, if $X$ is locally compact.

(ii) $Y$ and $\left(C(X, Y), \tau_{C O}\right)$ embed as closed subsets in $\left(\mathcal{P}, \tau_{C}\right)$.

Proof. (i) $x \mapsto\{x\}$ is a closed embedding of $X$ into $\left(C L(X), \tau_{F}\right)$. Let $y \in Y$ be fixed, and for each $A \in C L(X)$ define $f_{A} \in \mathcal{P}$ via $f_{A}(x)=y$ for all $x \in A$, then $\phi: A \mapsto f_{A}$ is an embedding of $\left(C L(X), \tau_{F}\right)$ into $\left(\mathcal{P}, \tau_{C}\right)$. Let $f \in \mathcal{P}$ be such that it has at least two distinct values $y_{1} \neq y_{2}$, and let $I_{1}, I_{2}$ be $Y$-open disjoint neighborhoods of $y_{1}, y_{2}$, respectively. If $X$ is locally compact, we can find $K_{1}, K_{2} \in K(X)$ such that $f\left(B \cap K_{1}\right) \subseteq I_{1}$, and $f\left(B \cap K_{2}\right) \subseteq I_{2}$, where $B=\operatorname{domf}$. Then

$$
f \in\left[K_{1}: I_{1}\right] \cap\left[K_{2}: I_{2}\right] \subseteq \mathcal{P} \backslash \phi(C L(X)),
$$

so $\phi(C L(X))$ is closed in $\left(\mathcal{P}, \tau_{C}\right)$.

(ii) Assigning to each $y \in Y$ the function $f_{y} \in C(X, Y)$, defined via $f_{y}(x)=y$ for all $x \in X$, sets up a closed embedding of $Y$ into $\left(C(X, Y), \tau_{C O}\right)$. Moreover, the identity map $i:\left(C(X, Y), \tau_{C O}\right) \rightarrow\left(\mathcal{P}, \tau_{C}\right)$ is clearly an embedding, and if $f \in \mathcal{P} \backslash i(C(X, Y))$ then there is $x \notin \operatorname{dom} f$, so

$$
f \in[\{x\}: \emptyset] \subseteq \mathcal{P} \backslash i(C(X, Y)) ;
$$

thus, $i(C(X, Y))$ is closed in $\left(\mathcal{P}, \tau_{C}\right)$.

Proposition 2.2. If $X$ is locally compact and $Y$ is Tychonoff, then $\left(\mathcal{P}, \tau_{C}\right)$ is a subspace of $\left(C L(X \times c Y), \tau_{F}\right)$.

Proof. To prove that $\tau_{C} \subseteq \tau_{F}$ on $\mathcal{P}$, let $U$ be an open set in $X$ and consider the $\tau_{C}$-open set $[U]$. If $f \in[U]$, then $(U \times c Y)^{-} \cap \mathcal{P}$ is a $\tau_{F}$-neighborhood of $f$ in $\mathcal{P}$ contained in $[U]$. Now, consider a $\tau_{C}$-open set $[K: I]$, where $K \in K(X)$ and $I$ is open in $Y$, and choose a $c Y$-open set $J$ with $I=J \cap Y$. It is easy to verify that

$$
[K: I]=\left((K \times(c Y \backslash J))^{c}\right)^{+} \cap \mathcal{P} .
$$

Conversely, let $U \subseteq X$ and $V \subseteq c Y$ be open, and $f \in(U \times V)^{-} \cap \mathcal{P}$. Then $(x, f(x)) \in U \times(V \cap Y)$ for some $x \in B:=\operatorname{dom} f$, so local compactness of $X$ and continuity of $f$ imply that there is an $X$-open neighborhood $O_{x}$ of $x$ with compact closure such that $\bar{O}_{x} \subseteq U$ and $f\left(\bar{O}_{x} \cap B\right) \subseteq V \cap Y$. Then

$$
f \in\left[O_{x}\right] \cap\left[\bar{O}_{x}: V \cap Y\right] \subseteq(U \times V)^{-} \cap \mathcal{P} .
$$

Finally, let $K$ be a compact set in $X \times c Y$ and $f \in\left(K^{c}\right)^{+}$. By local compactness, every compact set in $X \times c Y$ missing $f$ is contained in a finite union of product sets with compact factors missing $f$, so, without loss of generality, assume $K=K_{1} \times K_{2}$, where $K_{1} \in K(X), K_{2} \in K(c Y)$. It is easy to verify that $\Gamma(f) \subseteq\left(K_{1} \times K_{2}\right)^{c}$ if and only if $f\left(K_{1} \cap(\operatorname{domf})\right) \subseteq K_{2}^{c}$. In other words,

$$
\left[\left(K_{1} \times K_{2}\right)^{c}\right]^{+} \cap \mathcal{P}=\left[K_{1}:\left(K_{2}^{c}\right) \cap Y\right] .
$$

This implies, that $\left(K^{c}\right)^{+} \cap \mathcal{P} \in \tau_{C}$, so $\tau_{F}\left\lceil\mathcal{P} \subseteq \tau_{C}\right.$ indeed. 
Remark 2.3. Observe, that for $X$ locally compact and any $Y$, we can use the above argument to show that the Fell topology from $C L(X \times Y)$ on $\mathcal{P}$ is weaker than $\tau_{C}$. However, the converse is not true in general: indeed, consider $X=\{0\} \cup\left\{\frac{1}{n}: n \in \omega\right\}$, $Y=\omega$, both with the natural topology, and define $f_{n}:\left\{0, \frac{1}{n}\right\} \rightarrow Y$ via $f_{n}(0)=0$, $f_{n}\left(\frac{1}{n}\right)=n$ for each $n \in \omega$, and $f:\{0\} \rightarrow Y$ via $f(0)=0$. Then the sequence $\left\{f_{n}\right\}_{n} \tau_{F}$-converges to $f$, but fails to $\tau_{C}$-converge to $f$, since $f \in[X:\{0\}]$ but $f_{n} \notin[X:\{0\}]$ for all $n$.

It is not hard to show that $\left(\mathcal{P}, \tau_{C}\right)$ is always $T_{1}$; however, higher separation axioms impose some restrictions on $X$ and $Y$, as was shown in [Ho1, Ho2] for Tychonoff $X, Y$. We have the following more refined result on separation axioms with a new proof:

Proposition 2.4. The following are equivalent:

(i) $\left(\mathcal{P}, \tau_{C}\right)$ is Hausdorff (Tychonoff);

(ii) $X$ is locally compact and $Y$ is Hausdorff (Tychonoff).

Proof. (i) $\Rightarrow$ (ii) $Y$ has the relevant properties by Proposition 2.1(ii). Proposition 2.1(i) implies, that $\left(C L(X), \tau_{F}\right)$ is Hausdorff, so $X$ is locally compact by [Be, Proposition 5.1.2]. We can also give a direct proof of local compactness of $X$ : let $x \in X$ and $y_{1} \neq y_{2}$ be two different points of $Y$. Then $\left\{\left(x, y_{1}\right)\right\}$ and $\left\{\left(x, y_{2}\right)\right\}$ are two different elements of $\mathcal{P}$, so there are disjoint neighborhoods $H_{1}, H_{2}$ of $\left\{\left(x, y_{1}\right)\right\}$ and $\left\{\left(x, y_{2}\right)\right\}$, respectively in $\left(\mathcal{P}, \tau_{C}\right)$. We can assume that

$$
H_{1}=\left[U_{1}\right] \cap\left[K_{1}: I_{1}\right] \cap\left[L_{1}: \emptyset\right] \text { and } H_{2}=\left[U_{2}\right] \cap\left[K_{2}: I_{2}\right] \cap\left[L_{2}: \emptyset\right],
$$

where $U_{1}, U_{2}$ are nonempty $X$-open sets, $I_{1}, I_{2}$ are $Y$-open, $K_{1}, K_{2}, L_{1}, L_{2} \in K(X)$, $U_{1} \subseteq L_{1}^{c}$, and $U_{2} \subseteq L_{2}^{c}$. Since $\left\{\left(x, y_{1}\right)\right\} \in H_{1}$ and $\left\{\left(x, y_{2}\right)\right\} \in H_{2}$, we have $x \in$ $U_{1} \cap U_{2}$. Moreover, $U_{1} \cap U_{2} \subseteq K_{1} \cup K_{2}$, since otherwise, if $z \in U_{1} \cap U_{2} \backslash K_{1} \cup K_{2}$, then $\left\{\left(z, y_{1}\right)\right\} \in H_{1} \cap H_{2}$, a contradiction.

(ii) $\Rightarrow$ (i) Hausdorffness: let $f_{0}, f_{1} \in \mathcal{P}$ be distinct. If $\operatorname{dom} f_{0} \neq \operatorname{dom} f_{1}$, without loss of generality, take some $x \in \operatorname{dom} f_{0} \backslash \operatorname{dom} f_{1}$. Then there is an $X$-open $U$ and $K \in K(X)$ with $x \in U \subseteq K \subseteq X \backslash \operatorname{domf}_{1}$. It follows that $[U]$ and $[K: \emptyset]$ are disjoint $\tau_{C}$-open neighborhoods of $f_{0}$ and $f_{1}$, respectively. On the other hand, if $B=\operatorname{dom} f_{0}=\operatorname{dom} f_{1}$, then $f_{0}(x) \neq f_{1}(x)$ for some $x \in B$, so choosing disjoint $Y$-open neighborhoods $I_{0}, I_{1}$ of $f_{0}(x), f_{1}(x)$, respectively, as well as an $X$-open neighborhood $U$ of $x$ with compact closure such that $f_{i}(\bar{U} \cap B) \subseteq I_{i}$ for $i=0,1$, we have that $[U] \cap\left[\bar{U}: I_{0}\right]$ and $[U] \cap\left[\bar{U}: I_{1}\right]$ are disjoint $\tau_{C}$-open neighborhoods of $f_{0}$ and $f_{1}$, respectively.

Tychonoffness: $X \times \beta Y$ is Hausdorff and locally compact ( $\beta Y$ is the Cech-Stone compactification of $Y)$, so $\left(C L(X \times \beta Y), \tau_{F}\right)$ is Tychonoff [Be, Proposition 5.1.2], and, by our Proposition 2.2 , so is $\left(\mathcal{P}, \tau_{C}\right)$.

\section{3. Čech-Completeness and Related properties of $\left(\mathcal{P}, \tau_{C}\right)$}

From now on, $c Y$ is a fixed Hausdorff compactification of a Tychonoff space $Y$. Recall, that $Y$ is $\check{C}$ ech-complete [En], if $Y$ is $G_{\delta}$ in its compactification $c Y$. Also, $Y$ has a $G_{\delta}$-diagonal, if $\{(y, y): y \in Y\}$ is a $G_{\delta}$-set in $Y \times Y$, equivalently [Gr], if there exists a sequence $\left\{\mathcal{V}_{m}\right\}_{m}$ of $c Y$-open covers of $Y$ such that for each $y \in Y$, $\{y\}=Y \cap \bigcap_{m} \operatorname{St}\left(y, \mathcal{V}_{m}\right)$, where

$$
\operatorname{St}\left(y, \mathcal{V}_{m}\right)=\bigcup\left\{V \in \mathcal{V}_{m}: y \in V\right\} .
$$


We will say that $Y$ is a $p$-space [Arh, Gr], provided there is a feathering for $Y$, i.e. there is a sequence $\left\{\mathcal{V}_{m}\right\}_{m}$ of open covers of $Y$ in $c Y$ such that $\bigcap_{m} \operatorname{St}\left(y, \mathcal{V}_{m}\right) \subseteq Y$ for all $y \in Y$. Analogously, we can define cp-spaces, if we require $\bigcap_{m} \operatorname{St}\left(K, \mathcal{V}_{m}\right) \subseteq Y$ for all $K \in K(Y)$, where

$$
\operatorname{St}\left(K, \mathcal{V}_{m}\right)=\bigcup\left\{V \in \mathcal{V}_{m}: K \cap V \neq \emptyset\right\}
$$

It is easy to see that a Cech-complete space is a $c p$-space, which in turn is a $p$-space. On the other hand, a paracompact $p$-space is a $c p$-space: indeed, $Y$ is a paracompact $p$-space provided there is a metric space $Z$ and a perfect map $f: Y \rightarrow Z[\mathrm{Gr}]$. For each $m \geq 1$ consider the $Y$-cover

$$
\mathcal{V}_{m}^{\prime}=\left\{f^{-1}(U): U \text { open } Z \text {-ball of radius } \frac{1}{m}\right\}
$$

and define $\mathcal{V}_{m}=\left\{V \subseteq c Y\right.$ open $\left.: Y \cap V \in \mathcal{V}_{m}^{\prime}\right\}$. Take some $K \in K(Y)$, and assume there exists $p \in(c Y \backslash Y) \cap \bigcap_{m} \operatorname{St}\left(K, \mathcal{V}_{m}\right)$. Then for each $m$ we can find $V_{m} \in \mathcal{V}_{m}$ with $p \in V_{m}$ and $K \cap V_{m} \neq \emptyset$; let $y_{m} \in K \cap V_{m}$. The sequence $\left\{y_{m}\right\}_{m}$ has a cluster point $y \in K$, and by continuity of $f, f(y)$ is a cluster point of $\left\{f\left(y_{m}\right)\right\}_{m}$. The set $L=f^{-1}(f(y))$ is compact in $Y$ and hence in $c Y$ as well, thus, there exists a $c Y$-open $W$ containing $p$ such that $\bar{W}^{c Y} \subseteq c Y \backslash L$. Since $f$ is closed, $f\left(Y \cap \bar{W}^{c Y}\right)$ is a closed set missing $f(y)$. Note that each $f\left(Y \cap V_{m}\right)$ is a $Z$-open $\frac{1}{m}$-ball containing $f\left(y_{m}\right)$, and since $f(y)$ is a cluster point of $\left\{f\left(y_{m}\right)\right\}_{m}$, we can find $m$ so that $f\left(Y \cap V_{m}\right) \subseteq Z \backslash f\left(Y \cap \bar{W}^{c Y}\right)$. This leads to a contradiction, however, since $p \in W \cap V_{m}$, and so $Y \cap W \cap V_{m} \neq \emptyset$.

Let $X$ be a hemicompact space (i.e. in the family of all compact subspaces of $X$ ordered by inclusion, there exists a countable cofinal subfamily [En]). If $X$ is also locally compact, then there exists a sequence $\left\{C_{n}\right\}_{n}$ of compact sets covering $X$ such that $C_{n} \subseteq \operatorname{int} C_{n+1}$ (assume $C_{0}=\emptyset$ ). By Proposition 2.2, $\mathcal{P}$ with the Fell topology restricted from $C L(X \times c Y)$ coincides with $\tau_{C}$. Then $\mathcal{H}$, the closure of $\mathcal{P}$ in $\left(C L(X \times c Y), \tau_{F}\right)$, is locally compact and hence an open subspace of its Alexandroff one-point compactification $\alpha \mathcal{H}$.

Given a sequence $\left\{\mathcal{V}_{m}\right\}_{m}$ of $c Y$-open covers of $Y, m, n \in \omega$, a finite (possibly empty) collection $\mathcal{U}$ of nonempty $X$-open subsets of $C_{n+1}$, and $\varphi: \mathcal{U} \rightarrow \mathcal{V}_{m}$, the set

$$
\begin{aligned}
& H_{m, n}(\mathcal{U}, \varphi)= \\
& \mathcal{H} \cap\left(\left(\left(C_{n} \backslash \cup \mathcal{U}\right) \times c Y\right)^{c}\right)^{+} \cap \bigcap_{U \in \mathcal{U}}\left((U \times c Y)^{-} \cap\left((\bar{U} \times(c Y \backslash \varphi(U)))^{c}\right)^{+}\right)
\end{aligned}
$$

is open in $\alpha \mathcal{H}$. Let $\mathcal{H}_{m, n}$ be the collection of all these $H_{m, n}(\mathcal{U}, \varphi)$ 's. Note that $f \in \mathcal{P} \cap H_{m, n}(\mathcal{U}, \varphi)$ if and only if the following conditions are satisfied (denote $B=\operatorname{domf})$ :

(i) $B \cap C_{n} \subseteq \cup \mathcal{U}$,

(ii) $B \cap U \neq \emptyset$ for all $U \in \mathcal{U}$,

(iii) $f(B \cap \bar{U}) \subseteq \varphi(U)$ for all $U \in \mathcal{U}$.

We are now ready to prove some of our main results:

Theorem 3.1. Let $X$ be locally compact and hemicompact.

(i) If $Y$ is Čech-complete with a $G_{\delta}$-diagonal, then $\left(\mathcal{P}, \tau_{C}\right)$ is Čech-complete.

(ii) If $Y$ is a cp-space with a $G_{\delta}$-diagonal, then $\left(\mathcal{P}, \tau_{C}\right)$ is a p-space. 
Proof. (i) We will show that $\mathcal{P}$ is $G_{\delta}$ in $\alpha \mathcal{H}$ : let $\left\{\mathcal{V}_{m}\right\}_{m}$ be a sequence of covers of $Y$ consisting of $c Y$-open sets that witnesses both Cech-completeness and the $G_{\delta}$-diagonal property of $Y$. We claim that

$$
\mathcal{P}=\bigcap_{m, n \in \omega}\left(\bigcup \mathcal{H}_{m, n}\right) \text {. }
$$

Indeed, first take $f \in \mathcal{P}, m, n \in \omega$, and denote $B=d o m f$. If $B \cap C_{n}=\emptyset$, then $f \in H_{m, n}(\emptyset, \emptyset) \in \mathcal{H}_{m, n}$. If $B \cap C_{n} \neq \emptyset$, then by continuity of $f$, and compactness of $B \cap C_{n}$, there are finite families $\mathcal{U}$ of $X$-open subsets of $C_{n+1}$ and $\mathcal{W} \subseteq \mathcal{V}_{m}$ such that $B \cap C_{n} \subseteq \cup \mathcal{U}$, and for every $U \in \mathcal{U}, U \cap B \neq \emptyset$ and there is $W_{U} \in \mathcal{W}$ with $f(\bar{U} \cap B) \subseteq W_{U}$. Define $\varphi: \mathcal{U} \rightarrow \mathcal{V}_{m}$ via $\varphi(U)=W_{U}$ for every $U \in \mathcal{U}$. Then $f \in H_{m, n}(\mathcal{U}, \varphi) \in \mathcal{H}_{m, n}$.

Conversely, let $D \in \bigcap_{m, n \in \omega}\left(\bigcup \mathcal{H}_{m, n}\right)$. We will show that for every $x \in p_{X}(D)$, $D[x] \subseteq Y$ and $D[x]$ is a singleton, so $D$ is a closed graph of a function with a compact range; thus, $D \in \mathcal{P}$. Indeed, fix $m \in \omega$ and find $n \in \omega$ such that $x \in C_{n}$. There is a finite family $\mathcal{U}$ of nonempty $X$-open subsets of $C_{n+1}$, and $\varphi: \mathcal{U} \rightarrow \mathcal{V}_{m}$ such that $D \in H_{m, n}(\mathcal{U}, \varphi)$.

Then there is a $U \in \mathcal{U}$ containing $x$ (otherwise, $H_{m, n}(\mathcal{U}, \varphi) \subseteq\left((\{x\} \times c Y)^{c}\right)^{+} \cap \mathcal{H}$, and $x \notin p_{X}(D)$, so $D[x] \subseteq \varphi(U) \subseteq \cup \mathcal{V}_{m}$; thus, $D[x] \subseteq \bigcap_{m}\left(\cup \mathcal{V}_{m}\right)=Y$ by Čechcompleteness of $Y$. To prove that $D[x]$ is a singleton, suppose there are distinct $z, y \in D[x]$. Then, by the $G_{\delta}$-diagonal property, $z \in Y \cap \bigcap_{m} \operatorname{St}\left(y, \mathcal{V}_{m}\right)=\{y\}$, a contradiction.

(ii) Let $\left\{\mathcal{V}_{m}\right\}_{m}$ be a sequence of covers of $Y$ consisting of $c Y$-open sets that witness both the $c p$-space and $G_{\delta}$-diagonal property of $Y$. We claim that

$$
\left\{\mathcal{H}_{m, n}: m, n \in \omega\right\}
$$

is a feathering for $\mathcal{P}$ : to show that each $\mathcal{H}_{m, n}$ is an $\alpha \mathcal{H}$-open cover of $\mathcal{P}$, we can argue as in the first inclusion of $(*)$.

Now choose $f_{0} \in \mathcal{P}$ with $B_{0}=\operatorname{dom} f_{0}$, and consider $D \in \bigcap_{m, n} \operatorname{St}\left(f_{0}, \mathcal{H}_{m, n}\right)$. As in (i), it suffices to show that for every $x \in p_{X}(D), D[x] \subseteq Y$, and $D[x]$ is a singleton: let $n \in \omega$ be such that $x \in C_{n}$. For every $m \in \omega$ there is a finite family $\mathcal{U}$ of nonempty $X$-open subsets of $C_{n+1}$ and a $\varphi: \mathcal{U} \rightarrow \mathcal{V}_{m}$ such that $D, f_{0} \in H_{m, n}(\mathcal{U}, \varphi)$. Then $B_{0}$ intersects every element of $\mathcal{U}$, and there is a $U \in \mathcal{U}$ containing $x$, so we have

$$
D[x] \cup f_{0}\left(B_{0} \cap \bar{U}\right) \subseteq \varphi(U) \in \mathcal{V}_{m} .
$$

Consequently, by the $c p$-space property of $Y$,

$$
D[x] \subseteq \bigcap_{m} \operatorname{St}\left(f_{0}\left(B_{0} \cap C_{n+1}\right), \mathcal{V}_{m}\right) \subseteq Y .
$$

To prove that $D[x]$ is a singleton, we can use the argument from (i).

A sieve (cf. [Gr]) of $Y$ in $c Y$ is a pair $(G, T)$, where $(T,<)$ is an indexing tree of height $\omega$, and $G$ is a decreasing function from $T$ into the nonempty $c Y$-open sets (i.e. $t \leq t^{\prime}$ implies $\left.G(t) \supseteq G\left(t^{\prime}\right)\right)$ such that the sets corresponding to the initial level of $T$ cover $Y$, and for each $t \in T$,

$$
G(t) \cap Y=\bigcup\left\{G\left(t^{\prime}\right) \cap Y: t^{\prime} \text { is an immediate successor of } t\right\} .
$$

A thread of the sieve $(G, T)$ is the $G$-image of an infinite branch of $T$. If $Y$ is a $W_{\delta}$ subset of $c Y$ - i.e. there is a sieve of $Y$ in $c Y$ each thread of which intersects in a 
(nonempty) subset of $Y[\mathrm{CCN}]$ - then $Y$ is called sieve complete (the term was first used in [Mi1], but monotonically $\check{C}$ ech complete $[\mathrm{CCN}]$, and satisfying Condition $\mathcal{K}$ [WW] was also used; see also [Te]). Note that sieve completeness is independent of the compactification $c Y$ [CCN, Proposition 2.11]. It is known that sieve complete spaces are the continuous open images of Čech-complete spaces [WW], so Čechcomplete spaces are sieve complete; on the other hand, paracompact sieve complete spaces are Čech-complete [Mi1]. A space $Y$ has a $W_{\delta}$-diagonal [CCN], provided it has a sieve in $c Y$, such that if $\left\{V_{m}\right\}_{m}$ is any thread of it, and $y \in \bigcap_{m} V_{m} \cap Y$, then $\{y\}=\bigcap_{m} V_{m} \cap Y$. If $Y$ has a $G_{\delta}$-diagonal, it has a $W_{\delta}$-diagonal as well.

Theorem 3.2. Let $X$ be locally compact and hemicompact. If $Y$ is sieve complete with a $W_{\delta}$-diagonal, then $\left(\mathcal{P}, \tau_{C}\right)$ is sieve complete.

Proof. Let $(G, T)$ be a sieve of $Y$ in $c Y$ witnessing both sieve completeness and the $W_{\delta}$-diagonal property of $Y$. Let $T_{m}$ stand for the $m$-th level of $T$, and denote $\mathcal{V}_{m}=\left\{G(t): t \in T_{m}\right\}$ for all $m$. Inductively define a tree $S$, and a function $H$ from $S$ to the nonempty $\alpha \mathcal{H}$-open sets as follows: $S=\bigcup_{m} S_{m}$ will be a subtree of $\mathcal{P}^{<\omega}$, the tree of finite sequences of points of $\mathcal{P}$, where $S_{m} \subseteq \mathcal{P}^{m}$ is the $m$-th level of $S$. Let $S_{0}=\{\emptyset\}$, and put $H(\emptyset)=H_{0,0}(\emptyset, \emptyset)=\mathcal{H}$.

Let $S_{1}=\{(f): f \in \mathcal{P}\}$. To define $H(f)$ for $f \in \mathcal{P}$, first find the smallest $n$ for which $C_{n} \cap \operatorname{dom} f \neq \emptyset$, and using compactness of $C_{n} \cap \operatorname{dom} f$, get appropriate $\mathcal{U}, \varphi$ such that $f \in H_{1, n}(\mathcal{U}, \varphi)$. Finally, let $H(f)$ be an $\alpha \mathcal{H}$-open set such that

$$
f \in H(f) \subseteq \overline{H(f)}^{\alpha \mathcal{H}} \subseteq H_{1, n}(\mathcal{U}, \varphi) .
$$

Fix $m \geq 1$. Assume that $S_{m}$ has been defined, and for each $s \in S_{m}, H(s)$ has been chosen so that $\overline{H(s)}^{\alpha \mathcal{H}} \subseteq H\left(s^{\prime}\right) \cap H_{m, n_{s}}\left(\mathcal{U}_{s}, \varphi_{s}\right)$ for some nonempty $\mathcal{U}_{s}, \varphi_{s}$, where $s^{\prime} \in S_{m-1}$ is the predecessor of $s$, and $n_{s}=n_{s^{\prime}}+1$. Given $s \in S_{m},(s, f) \in \mathcal{P}^{m+1}$ will be an immediate successor of $s$ in $S$, if $f \in \mathcal{P} \cap H(s)$, so

$$
S_{m+1}=\left\{(s, f): s \in S_{m}, f \in \mathcal{P} \cap H(s)\right\} .
$$

Let $(s, f) \in S_{m+1}$, and $B=d o m f$. Then $f \in H_{m, n_{s}}\left(\mathcal{U}_{s}, \varphi_{s}\right)$, so $B \cap C_{n_{s}} \subseteq \bigcup \mathcal{U}_{s}$, and for all $U \in \mathcal{U}_{s}, U \subseteq C_{n_{s}+1}, B \cap U \neq \emptyset$, and $f(B \cap \bar{U}) \subseteq \varphi_{s}(U)$. Fix $U \in \mathcal{U}_{s}$; then $\varphi_{s}(U)=G(t)$ for some $t \in T_{m}$. Consider the collection

$$
\mathcal{W}(U)=\left\{G\left(t^{\prime}\right) \in \mathcal{V}_{m+1}: t^{\prime} \in T_{m+1}, t^{\prime}>t\right\},
$$

for which $Y \cap \varphi_{s}(U)=\bigcup\{Y \cap W: W \in \mathcal{W}(U)\}$, and $W \subseteq \varphi_{s}(U)$ for all $W \in \mathcal{W}(U)$. Since $\bigcup_{U \in \mathcal{U}_{s}} \mathcal{W}(U)$ is a $c Y$-open cover of $f\left(B \cap C_{n_{s}}\right)$, each $x \in B \cap C_{n_{s}}$ has an $X$-open neighborhood $O_{x}$ so that $\overline{O_{x}} \subseteq U$ for some $U \in \mathcal{U}_{s}$, and $f\left(B \cap \overline{O_{x}}\right) \subseteq W$ for some $W \in \mathcal{W}(U)$.

By compactness of $B \cap C_{n_{s}}$, we can find $x_{0}, \ldots, x_{p} \in B \cap C_{n_{s}}$ such that $B \cap C_{n_{s}} \subseteq$ $\bigcup_{i \leq p} O_{x_{i}}$, and for all $i \leq p$, there is some $W_{i} \in \bigcup_{U \in \mathcal{U}_{s}} \mathcal{W}(U)$ with $f\left(B \cap \overline{O_{x_{i}}}\right) \subseteq W_{i}$. Denote

$$
C=C_{n_{s}+1} \backslash \bigcup_{i \leq p} O_{x_{i}},
$$

and let $\mathcal{N}=\emptyset$, if $B \cap C=\emptyset$. If $B \cap C \neq \emptyset$ then, because $\mathcal{V}_{m+1}$ covers $Y$, each $z \in B \cap C$ has an $X$-open neighborhood $N_{z}$ such that $\overline{N_{z}} \subseteq \operatorname{int} C_{n_{s}+2} \backslash C_{n_{s}}$, and $f\left(B \cap \overline{N_{z}}\right) \subseteq V$ for some $V \in \mathcal{V}_{m+1}$. By compactness of $B \cap C$, we can find $z_{0}, \ldots, z_{r} \in B \cap C$ such that $B \cap C \subseteq \bigcup_{j \leq r} N_{j}$, and for all $j \leq r$ there is some $V_{j} \in \mathcal{V}_{m+1}$ with $f\left(B \cap \overline{N_{z_{j}}}\right) \subseteq V_{j}$; put $\mathcal{N}=\left\{N_{z_{j}}: j \leq r\right\}$ 
Define $\mathcal{U}_{(s, f)}=\left\{O_{x_{i}}: i \leq p\right\} \cup \mathcal{N}$, and

$$
\varphi_{(s, f)}(A)= \begin{cases}W_{i}, & \text { if } A=O_{x_{i}} \text { for some } i \leq p, \\ V_{j}, & \text { if } A=N_{z_{j}} \text { for some } j \leq r .\end{cases}
$$

It is clear from the above construction, that $f \in H_{m+1, n_{s}+1}\left(\mathcal{U}_{(s, f)}, \varphi_{(s, f)}\right)$, so we can find an $\alpha \mathcal{H}$-open set $H(s, f)$ such that putting $n_{(s, f)}=n_{s}+1$ we have

$$
f \in H(s, f) \subseteq \overline{H(s, f)}^{\alpha \mathcal{H}} \subseteq H(s) \cap H_{m+1, n_{(s, f)}}\left(\mathcal{U}_{(s, f)}, \varphi_{(s, f)}\right) .
$$

Then $H(s, f) \subseteq H(s)$, and $\mathcal{P} \cap H(s)=\bigcup\{\mathcal{P} \cap H(s, f): f \in \mathcal{P} \cap H(s)\}$, so $(H, S)$ is a sieve of $\mathcal{P}$ in $\alpha \mathcal{H}$.

Finally, let $\left\{H\left(s_{m}\right): m \geq 1\right\}$ be a thread in $(H, S)$, where for all $m, s_{m} \in S_{m}$, and $s_{m+1}$ is an immediate successor of $s_{m}$ in $S$. Let $\mathcal{U}_{s_{m}}$ be a finite collection of $X$-open sets, and $\varphi_{s_{m}}: \mathcal{U}_{s_{m}} \rightarrow \mathcal{V}_{m}$ such that

$$
{\overline{H\left(s_{m}\right)}}^{\alpha \mathcal{H}} \subseteq H\left(s_{m-1}\right) \cap H_{m, n_{s_{m}}}\left(\mathcal{U}_{s_{m}}, \varphi_{s_{m}}\right) .
$$

Since $\alpha \mathcal{H}$ is compact, then $\bigcap_{m} H\left(s_{m}\right) \neq \emptyset$, so we can choose $D \in \bigcap_{m} H\left(s_{m}\right)$, and $x \in p_{X}(D)$. Find the first $m$ with $x \in C_{n_{s_{m}}}$. Then the set

$$
T_{k}^{\prime}=\left\{t \in T_{k}: \exists U \in \mathcal{U}_{s_{k}} \text { with } x \in U \text { and } G(t)=\varphi_{s_{k}}(U)\right\}
$$

is nonempty for each $k \geq m$. Moreover, if $t \in T_{k}^{\prime}$ for some $k>m$, and $U \in \mathcal{U}_{s_{k}}$ is such that $G(t)=\varphi_{s_{k}}(U)$, then by the definition of $\mathcal{U}_{s_{k}}$, either $U$ is disjoint from $C_{s_{k-1}}$, which is not the case, since $x \in C_{s_{m}} \cap U \subseteq C_{s_{k-1}} \cap U$, or there exists a $U^{\prime} \in \mathcal{U}_{s_{k-1}}$ with $U \subseteq U^{\prime}$ and $G(t) \in \mathcal{W}\left(U^{\prime}\right)$. Then there is $t^{\prime} \in T_{k-1}$ with $t^{\prime}<t$, and $G\left(t^{\prime}\right)=\varphi_{s_{k-1}}\left(U^{\prime}\right)$; thus, $t^{\prime} \in T_{k-1}^{\prime}$. It means that $T^{\prime}=\bigcup_{k>m} T_{k}^{\prime}$ is a subtree of $T$ of height $\omega$, which is clearly finite splitting (i.e. each node in $T^{\prime}$ has only finitely many immediate successors - see $[\mathrm{Ke}])$. By König's Lemma, $T^{\prime}$ has an infinite branch

$$
t_{m}<\cdots<t_{k}<t_{k+1}<\cdots
$$

where $t_{k} \in T_{k}^{\prime}$ for each $k \geq m$. Let $U_{k} \in \mathcal{U}_{s_{k}}$ be such that $\varphi_{s_{k}}\left(U_{k}\right)=G\left(t_{k}\right)$. Then $x \in U_{k}$, and $D[x] \subseteq \varphi_{s_{k}}\left(U_{k}\right)$ for all $k \geq m$, so $\left\{\varphi_{s_{k}}\left(U_{k}\right): k \geq m\right\}$ is part of a thread of $(G, T)$ containing $D(x)$. This thread is intersecting in a singleton $y \in Y$, since $Y$ is sieve complete with a $W_{\delta}$-diagonal. It follows, that $y=D(x)$; thus, $D \in \mathcal{P}$.

A space $Y$ is a $q$-space $[\mathrm{Mi} 2, \mathrm{Ch}]$, if for each $y \in Y$ there is a sequence $\left\{U_{n}\right\}_{n}$ of neighborhoods of $y$ such that whenever $y_{n} \in U_{n}$, the sequence $\left\{y_{n}\right\}_{n}$ has a cluster point. Note that sieve complete, as well as, $p$-spaces are $q$-spaces.

Proposition 3.3. Let $Y$ be a non-countably compact space. If $\left(\mathcal{P}, \tau_{C}\right)$ is a q-space, then $X$ is hemicompact.

Proof. Let $y \in Y$, and define $f(x)=y$ for each $x \in X$. Let $\mathcal{U}_{n}=\bigcap_{p \in P_{n}}\left[U_{n}^{p}\right] \cap$ $\bigcap_{r \in R_{n}}\left[K_{n}^{r}: I_{n}^{r}\right]$ (where $P_{n}, R_{n}$ are finite sets) be a sequence of $\tau_{C}$-neighborhoods of $f$ satisfying the $q$-space property at $f$.

For every $p \in P_{n}$ choose $x_{n}^{p} \in U_{n}^{p}$ and put $K_{n}=\left\{x_{n}^{p}: p \in P_{n}\right\} \cup \bigcup_{r \in R_{n}} K_{n}^{r}$. Then $K_{n}$ is compact for every $n$, further, $\left\{K_{n}\right\}_{n}$ is a countable cofinal subfamily of $K(X)$ : otherwise, let $K \in K(X)$ be such that for every $n \in \omega$ there is $k_{n} \in K \backslash K_{n}$. Let $\left\{y_{n}\right\}_{n}$ be a sequence without a cluster point in $Y$. Define $f_{n}: K_{n} \cup\left\{k_{n}\right\} \rightarrow Y$ via $f_{n}(x)=f(x)$ for $x \in K_{n}$, and $f_{n}\left(k_{n}\right)=y_{n}$. It follows, that the sequence $f_{n} \in \mathcal{U}_{n}$ clusters in some $h \in \mathcal{P}$. The set $h(K \cap \operatorname{dom} h)$ is compact, so there is an 
open $I \subseteq Y$ and $n_{0} \in \omega$ such that $h(K \cap \operatorname{dom} h) \subseteq I$, and $y_{n} \notin I$ for every $n \geq n_{0}$. Then $[K: I]$ is a $\tau_{C}$-neighborhood of $h$, and for every $n \geq n_{0}, f_{n} \notin[K: I]$, which is a contradiction.

Remark 3.4. If $Y$ is countably compact, the above proposition may fail: indeed, if $Y=\{0,1\}$ and $X$ is an uncountable discrete space, then $\left(\mathcal{P}, \tau_{C}\right)$ is a closed subspace of $\left(C L(X \times Y), \tau_{F}\right)$, so it is Cech-complete, but $X$ is not hemicompact.

Theorem 3.5. Let $Y$ be a non-countably compact space with a $G_{\delta}$-diagonal ( $W_{\delta}$ diagonal). Then the following are equivalent:

(i) $\left(\mathcal{P}, \tau_{C}\right)$ is Čech-complete (sieve complete);

(ii) $X$ is locally compact, Lindelöf, and $Y$ is Čech-complete (sieve complete).

Proof. (i) $\Rightarrow$ (ii) By Proposition 2.4, $X$ is locally compact, by Proposition $3.3, X$ is hemicompact, and hence Lindelöf. Moreover, $Y$ is Čech-complete (sieve complete) by Proposition 2.1(ii).

(ii) $\Rightarrow$ (i) See Theorem 3.1(i), and Theorem 3.2.

Theorem 3.6. Let $Y$ be a non-compact, paracompact space with a $G_{\delta}$-diagonal. Then the following are equivalent:

(i) $\left(\mathcal{P}, \tau_{C}\right)$ is a p-space;

(ii) $X$ is locally compact, Lindelöf, and $Y$ is metrizable.

Proof. (i) $\Rightarrow$ (ii) By Proposition 2.4, $X$ is locally compact, by Proposition 3.3, $X$ is hemicompact, and hence Lindelöf. Moreover, $Y$ is a $p$-space by Proposition 2.1(ii), and a paracompact $p$-space with a $G_{\delta}$-diagonal is metrizable [Gr] .

(ii) $\Rightarrow$ (i) See Theorem 3.1(ii).

The following theorem is an extension of [Ho2, Theorem 3.3] (for another proof see $[\mathrm{NZ}])$ :

Theorem 3.7. The following are equivalent:

(i) $\left(\mathcal{P}, \tau_{C}\right)$ is completely metrizable;

(ii) $X$ is hemicompact, metrizable, and $Y$ is completely metrizable.

Proof. (i) $\Rightarrow$ (ii) $Y$ is completely metrizable by Proposition 2.1(ii); further, metrizability of $\left(\mathcal{P}, \tau_{C}\right)$ implies metrizability of $\left(C L(X), \tau_{F}\right)$ (see Proposition 2.1(i)), which in turn is equivalent to hemicompactness and metrizability of $X$ [Be, Theorem 5.1.5].

(ii) $\Rightarrow(\mathrm{i})\left(\mathcal{P}, \tau_{C}\right)$ is metrizable by [Ho2, Theorem 2.4], and Cech-complete by our Theorem 3.1, hence it is completely metrizable [En, Theorem 4.3.26].

Finally, using the argument of [Ho2, Theorem 3.4], we can fully characterize Polishness of $\left(\mathcal{P}, \tau_{C}\right)$ :

Theorem 3.8. The following are equivalent:

(i) $\left(\mathcal{P}, \tau_{C}\right)$ is Polish;

(ii) $X$ is hemicompact, metrizable, and $Y$ is Polish. 


\section{4. ČECh-COMPleteness AND RELATED PROPERTIES OF THE COMPACT-OPEN TOPOLOGY ON $C(X, Y)$}

Properties of the compact-open topology $\tau_{C O}$ are well established for $Y=\mathbb{R}$, or more generally for a locally convex completely metrizable $Y$ (cf. [MN1, En], or more recently [GRe]). This restriction on $Y$ is usually required to use some extension theorem (Tietze, Dugundji) in order to obtain the desired result. As we have shown in previous sections, one can get by in $\left(\mathcal{P}, \tau_{C}\right)$ without extension theorems, and with considerably more general $Y$. On the other side, $\left(C(X, Y), \tau_{C O}\right)$ embeds as a closed subset in $\left(\mathcal{P}, \tau_{C}\right)$, which leads to results on $\tau_{C O}$ with less restricted $Y$. For theorems of this general nature see e.g. [Are], [Mi3], [OM] or [SM].

To be more specific, note that if $X$ is a hemicompact $k$-space and $Y$ is completely metrizable, then $\left(C(X, Y), \tau_{C O}\right)$ is completely metrizable [MN1], and hence Cechcomplete, sieve complete, as well as a $p$-space. As a corollary to Theorem 3.2 (resp. Theorem 3.1), we can generalize this result to a sieve complete (resp. Čechcomplete, cp-space) $Y$ with a $W_{\delta}$-diagonal (resp. $G_{\delta}$-diagonal). For another proof see [HZ2]:

Theorem 4.1. Let $X$ be a hemicompact $k$-space, and $Y$ be sieve complete (Čechcomplete, cp-space, resp.) with a $W_{\delta}$-diagonal $\left(G_{\delta}\right.$-diagonal, resp. $)$.

Then $\left(C(X, Y), \tau_{C O}\right)$ is sieve complete (Čech-complete, $p$-space, resp.).

Proof. Let $\left\{C_{n}\right\}_{n}$ be a cofinal collection in $K(X)$, and $Z=\bigoplus_{n} C_{n}$ the topological sum of the $C_{n}$ 's. Then $Z$ is hemicompact, locally compact and, since $X$ is a $k$-space, the natural mapping $\psi: Z \rightarrow X$ is compact-covering and quotient. Consequently, the map $\psi^{*}:\left(C(X, Y), \tau_{C O}\right) \rightarrow\left(C(Z, Y), \tau_{C O}\right)$, defined via

$$
\psi^{*}(f)=f \circ \psi, \text { for all } f \in C(X, Y),
$$

is a closed embedding [MN1, Corollary 2.2.8(b), and Theorem 2.2.10]. It follows from Theorem 3.2 (Theorem 3.1, resp.), and Proposition 2.1(ii), that $\left(C(Z, Y), \tau_{C O}\right)$ is sieve complete (Čech-complete, $p$-space, resp.), as is $\left(C(X, Y), \tau_{C O}\right)$, which sits in $\left(C(Z, Y), \tau_{C O}\right)$ as a closed subspace.

\section{Paracompactness and Related properties of $\left(\mathcal{P}, \tau_{C}\right)$}

Since a paracompact $p$-space with a $G_{\delta}$-diagonal is metrizable $[\mathrm{Gr}]$, we have

Lemma 5.1. A locally compact, Lindelöf space with a $G_{\delta}$-diagonal is metrizable.

Theorem 5.2. Let $Y$ be a Polish space. The following are equivalent:

(i) $\left(\mathcal{P}, \tau_{C}\right)$ is Polish;

(ii) $\left(\mathcal{P}, \tau_{C}\right)$ is Lindelöf;

(iii) $X$ is hemicompact, and metrizable.

Proof. For (iii) $\Leftrightarrow\left(\right.$ i) see Theorem 3.8. As for (ii) $\Rightarrow\left(\right.$ iii), observe that $\left(\mathcal{P}, \tau_{C}\right)$ Hausdorff implies $X$ is locally compact (Proposition 2.4), so by Proposition 2.1(i), $\left(C L(X), \tau_{F}\right)$ is Lindelöf, and so is $X$. This in turn implies that $\left(C(X, Y), \tau_{C O}\right)$ is metrizable [MN1, Exercise 4.9.1] and, by Proposition 2.1(ii), Lindelöf; thus, 2nd 
countable. Then, by [MN1, Exercise 4.9.7(a)], $X$ is an $\aleph_{0}$-space (i.e. it has a countable $k$-network $[\mathrm{Gr}])$. Finally, since an $\aleph_{0}$-space has a $G_{\delta}$-diagonal, $X$ is metrizable by Lemma 5.1.

Proposition 5.3. Let $Y$ be sieve complete (Čech-complete, cp-space, resp.) with a $W_{\delta}$-diagonal $\left(G_{\delta}\right.$-diagonal, resp.). If $\left(\mathcal{P}, \tau_{C}\right)$ is normal, then $\left(\mathcal{P}, \tau_{C}\right)$ is sieve complete (Čech-complete, a p-space, resp.).

Proof. $\left(C L(X), \tau_{F}\right)$ is normal since, by Proposition 2.4 and Proposition 2.1(i), it embeds as a closed subset in $\left(\mathcal{P}, \tau_{C}\right)$, so $X$ is locally compact and hemicompact $\left[\right.$ HLP]. By Theorem 3.2 (Theorem 3.1), $\left(\mathcal{P}, \tau_{C}\right.$ ) is sieve complete (Čech-complete, a $p$-space, resp.).

If we restrict our attention to spaces with $G_{\delta}$-diagonals, it is possible to extend Theorem 5.2:

Theorem 5.4. Let $X$ have a $G_{\delta}$-diagonal, and $Y$ be a non-compact Polish space. Then the following are equivalent:

(i) $\left(\mathcal{P}, \tau_{C}\right)$ is Polish;

(ii) $\left(\mathcal{P}, \tau_{C}\right)$ is normal;

(iii) $X$ is locally compact, and Lindelöf.

Proof. (iii) $\Rightarrow$ (i) follows from Lemma 5.1, and Theorem 3.8.

(ii) $\Rightarrow$ (iii) follows from Proposition 5.3, and Theorem 3.5.

Proposition 5.5. $\left(\mathcal{P}, \tau_{C}\right)$ is paracompact if and only if it is Lindelöf, if either of the following is satisfied:

(i) $X$ has a $G_{\delta}$-diagonal and $Y$ is 2nd countable;

(ii) $X, Y$ are separable.

Proof. The nontrivial part is to prove that paracompactness implies Lindelöfness. Paracompactness of $\left(\mathcal{P}, \tau_{C}\right)$ implies paracompactness of $\left(C L(X), \tau_{F}\right)$ (by Proposition 2.4 and Proposition 2.1(i)), so $X$ is locally compact and Lindelöf [HLP].

Then by Lemma 5.1, (i) implies (ii), so it suffices to prove Lindelöfness of $\left(\mathcal{P}, \tau_{C}\right)$ for (ii): let $D_{X}, D_{Y}$ be countable dense sets in $X$ and $Y$, respectively. We will be done if we show that $\left(\mathcal{P}, \tau_{C}\right)$ is separable: by [HZ1, Proposition 2.1(iii)], the collection of sets

$$
\left[K_{0}: \emptyset\right] \cap \bigcap_{i \leq n}\left[U_{i}\right] \cap\left[\overline{U_{i}}: I_{i}\right]
$$

with $n \geq 1, K_{0}, \overline{U_{i}} \in K(X), \emptyset \neq U_{i} \subseteq X$ open, $K_{0}, U_{i}$ pairwise disjoint for $i \leq n$ and $\emptyset \neq I_{i} \subseteq Y$ open $(i \leq n)$, forms a $\pi$-base for $\tau_{C}$. Using this $\pi$-base, it is not hard to verify that the collection of continuous partial maps with values in $D_{Y}$, the domains of which run over the finite subsets of $D_{X}$, is dense (and countable) in $\left(\mathcal{P}, \tau_{C}\right)$.

Remark 5.6. Note that paracompactness of $\left(\mathcal{P}, \tau_{C}\right)$ is not equivalent to Lindelöfness and local compactness of $X$ (as Theorem 5.4 would suggest), since otherwise (comparing Theorem 5.2 and Proposition 5.5(ii)), a separable, locally compact, Lindelöf space would be metrizable (which is not the case, just consider $\beta \omega$ ). The following results suggest rather, that paracompactness of $\left(\mathcal{P}, \tau_{C}\right)$ could be closer to metrizability of $\left(\mathcal{P}, \tau_{C}\right)$. 
Proposition 5.7. Let $Y$ be a p-space with a $G_{\delta}$-diagonal. The following are equivalent:

(i) $\left(\mathcal{P}, \tau_{C}\right)$ is paracompact;

(ii) $\left(\mathcal{P}, \tau_{C}\right)$ is a paracompact $p$-space;

Proof. (i) $\Rightarrow$ (ii) follows from Proposition 5.3, since $Y$ is paracompact by Proposition 2.1 (ii), and a paracompact $p$-space is a $c p$-space.

Theorem 5.8. Let $Y$ be a p-space. The following are equivalent:

(i) $\left(\mathcal{P}, \tau_{C}\right)$ is paracompact with a $G_{\delta}$-diagonal;

(ii) $\left(\mathcal{P}, \tau_{C}\right)$ is metrizable;

(iii) $X$ is hemicompact metrizable, and $Y$ is metrizable.

Proof. (i) $\Rightarrow$ (ii) It follows from Proposition 2.1(ii), that $Y$ has a $G_{\delta^{-}}$-diagonal, so by Proposition 5.7, $\left(\mathcal{P}, \tau_{C}\right)$ is a paracompact $p$-space with a $G_{\delta^{-}}$-diagonal, and hence, metrizable [Gr]. The remaining implications follow from [Ho2, Theorem 2.4].

The following lemma is needed to prove our last characterization of paracompactness of $\left(\mathcal{P}, \tau_{C}\right)$, but it may be of independent interest:

Lemma 5.9. Let $X, Y$ be such that partial continuous functions with closed domains are continuously extendable over $X$; moreover, suppose that there exists an open base $\mathcal{V}$ for $Y$ closed under finite intersections such that for each nonempty $K \in K(X)$ and $V \in \mathcal{V}$, every function $f \in C(K, V)$ is extendable to some $f^{*} \in C(X, V)$.

Then the set-valued function $\psi:\left(\mathcal{P}, \tau_{C}\right) \rightrightarrows\left(C(X, Y), \tau_{C O}\right)$ defined via

$$
\psi(f)=\left\{g \in C(X, Y): g\left\lceil_{\operatorname{dom} f}=f\right\}\right.
$$

is lower semicontinuous.

Proof. The restriction mapping

$$
\eta:\left(C L(X), \tau_{F}\right) \times\left(C(X, Y), \tau_{C O}\right) \rightarrow\left(\mathcal{P}, \tau_{C}\right),
$$

defined via $\eta(B, f)=f \uparrow_{B}$, is an open mapping (see [HZ1, Proposition 3.3]). It suffices to notice that $\psi^{-1}(\mathbf{V})=\eta(C L(X) \times \mathbf{V}) \in \tau_{C}$ for any $\mathbf{V} \in \tau_{C O}$.

Theorem 5.10. Let $Y$ be a Frèchet space. The following are equivalent:

(i) $\left(\mathcal{P}, \tau_{C}\right)$ is paracompact;

(ii) $X$ is locally compact, Lindelöf, and there exists a continuous mapping $\varphi:\left(\mathcal{P}, \tau_{C}\right) \rightarrow\left(C(X, Y), \tau_{C O}\right)$ such that $\varphi(f)$ is a continuous extension of $f \in \mathcal{P}$;

(iii) $X$ is locally compact, Lindelöf, and $\left(\mathcal{P}, \tau_{C}\right)$ embeds as a closed subset in $\left(C L(X), \tau_{F}\right) \times\left(C(X, Y), \tau_{C O}\right)$.

Proof. (i) $\Rightarrow$ (ii) $X$ is locally compact and Lindelöf by [HLP], since $\left(C L(X), \tau_{F}\right)$ is paracompact by Proposition 2.4, and Proposition 2.1(i). Now use Lemma 5.9 along with Michael's Selection Theorem, as in the proof of [Ho2, Theorem 4.1], to finish the proof.

(ii) $\Rightarrow$ (iii) See the proof of [Ho2, Theorem 4.2].

(iii) $\Rightarrow$ (i) If $X$ is locally compact and Lindelöf, then $\left(C L(X), \tau_{F}\right)$ is locally compact, paracompact [HLP] and $\left(C(X, Y), \tau_{C O}\right)$ is metrizable [MN1]; hence, the product $\left(C L(X), \tau_{F}\right) \times\left(C(X, Y), \tau_{C O}\right)$ is paracompact [En, Exercise 5.5.5.(c)]. 
Paracompactness and normality have been studied for the Fell topology [HLP], as well as for the compact-open topology $[\mathrm{Po}],[\mathrm{OM}]$. For the generalized compactopen topology we only have the above partial results, so we ask:

Problem 5.11. Characterize paracompactness (normality) of $\left(\mathcal{P}, \tau_{C}\right)$ in terms of $X$ and $Y$. In particular, is paracompactness (normality) of $\left(\mathcal{P}, \tau_{C}\right)$ equivalent to its metrizability for some reasonable $Y($ say $Y=\mathbb{R})$ ?

\section{ACKNOWLEDGEMENT}

The authors are grateful for the helpful comments of the referees (especially concerning Theorem 3.2).

\section{REFERENCES}

[AA] A. Abd-Allah, Partial maps in algebra and topology, Ph.D. thesis, University of Wales, 1979.

[AB] A. Abd-Allah and R. Brown, A compact-open topology on partial maps with open domains, J. London Math. Soc. 21 (1980), 480-486.

[Are] R. Arens, A topology of transformations, Annals of Math. 47 (1946), 480-495.

[Arh] A. V. Arhangel'skiı̌ $A$ class of spaces containing all metric and locally compact spaces, Mat. Sb. 67 (1970), 55-58.

[Ba] J. Back, Concepts of similarity for utility functions, J. of Math. Economics 1 (1986), 721-727.

[BB] P. I. Booth and R. Brown, Spaces of partial maps, fibered mapping spaces and the compact open topology, Topology Appl. 8 (1978), 181-195.

[BC] P. Brandi and R. Ceppitelli, Existence, uniqueness and continuous dependence for hereditary differential equations, J. Diff. Equations 81 (1989), 317-339.

[BCH1] P. Brandi, R. Ceppitelli and L. Holá, Topological properties of a new graph topology, J. Convex Anal. 5 (1998), 1-12.

[BCH2] P. Brandi, R. Ceppitelli and L. Holá, Kuratowski convergence on compacta and Hausdorff metric convergence on compacta, Comment. Math. Univ. Carolin. 40 (1999), 309-318.

[Be] G. Beer, Topologies on Closed and Closed Convex Sets, Kluwer, Dordrecht, 1993.

[CCN] J. Chaber, M. M. Čban and K. Nagami, On monotonic generalizations of Moore spaces, Čech complete spaces and p-spaces, Fund. Math. 84 (1974), 107-119.

[Ch] T. Chiba, On q-spaces, Proc. Japan Acad. 45 (1969), 453-456.

[DN1] A. Di Concilio and S. A. Naimpally, Proximal set-open topologies and partial maps, Acta Math. Hung. 88 (2000), 227-237.

[DN2] A. Di Concilio and S. A. Naimpally, Function space topologies on (partial) maps, Recent Progress in Function Spaces, Quaderni di Matematica 3, 1998.

[En] R. Engelking, General Topology, Helderman, Berlin, 1989.

[Fe] J. Fell, A Hausdorff topology for the closed subsets of locally compact non-Hausdorff space, Proc. Amer. Math. Soc. 13 (1962), 472-476.

[Fi] V. V. Filippov, Basic topological structures of the theory of ordinary differential equations, Topology in Nonlinear Analysis, Banach Centrum Publications 35 (1996), 171-192.

[Gr] G. Gruenhage, Generalized metric spaces, in Handbook of Set-Theoretic Topology, NorthHolland, Amsterdam, 1984.

[GRe] P. M. Gartside and E. A. Reznichenko, Near metric properties of function spaces, Fund. Math. 164 (2000), 97-114.

[HLP] L. Holá, S. Levi and J. Pelant, Normality and paracompactness of the Fell topology, Proc. Amer. Math. Soc. 127 (1999), 2193-2197.

[HM] D. Holý and L. Matejička, C-upper semicontinuous and $C^{*}$ - upper semicontinuous multifunctions, Tatra Mt. Math. Publ. 34 (2006), 159-165.

[Ho1] L. Holá, Uniformizability of the generalized compact-open topology, Tatra Mt. Math. Publ. 14 (1998), 219-224.

[Ho2] L. Holá, Complete metrizability of generalized compact-open topology, Topology Appl. 91 (1999), 159-167. 
[HZ1] L. Holá and L. Zsilinszky, Completeness properties of the generalized compact-open topology on partial functions with closed domains, Topology Appl. 110 (2001), 303-321.

[HZ2] L. Holá and L. Zsilinszky, Vietoris topology on partial maps with compact domains, Topology Appl., to appear.

[Ke] A. S. Kechris Classical Descriptive Set Theory, Springer, New York, 1994.

[KS] H. P. Künzi and L. B. Shapiro, On simultaneous extension of continuous partial functions, Proc. Amer. Math. Soc. 125 (1997), 1853-1859.

[KT] E. Klein and A. Thompson, Theory of Correspondences, Wiley, New York, 1975.

[Ku1] K. Kuratowski, Sur l'espace des fonctions partielles, Ann. Mat. Pura Appl. 40 (1955), 61-67.

[Ku2] K. Kuratowski, Sur une méthode de métrisation complète de certains espaces d'ensembles compacts, Fund. Math. 43 (1956), 114-138.

[La] H.J. Langen, Convergence of dynamic programming models, Mathematics of Operations Research 6 (1981), 493-512.

[Mi1] E. Michael, Complete spaces and tri-quotient maps, Illinois J. Math. 21 (1977), 716-733.

[Mi2] E. Michael, A note on closed maps and compact sets, Israel J. Math. 2 (1964), 173-176.

[Mi3] E. Michael, $\aleph_{0}$-spaces, J. Math. Mech. 15 (1966), 983-1002.

[MN1] R. A. McCoy and I. Ntantu, Topological properties of spaces of continuous functions, Springer-Verlag, Berlin, 1988.

[MN2] R. A. McCoy and I. Ntantu, Completeness properties of function spaces, Topology Appl. 22 (1986), 191-206.

[NZ] P. J. Nyikos and L. Zsilinszky, Strong $\alpha$-favorability of the (generalized) compact-open topology, Atti Sem. Mat. Fis. Univ. Modena 51 (2003), 1-8.

[OM] P. O'Meara, On paracompactness in function spaces with the compact-open topology, Proc. Am. Math. Soc.29 (1971), 183-189.

[Po] R. Pol, Normality in function spaces, Fund. Math. 84 (1974), 145-155.

[Se] G. R. Sell, On the Fundamental Theory of Ordinary Differential Equations, J. Differential Equations 1 (1965), 371-392.

[SM] N. Shimane and T. Mizokami, On the embedding and developability of mapping spaces with compact-open topology, Topology Proc. 24 (1999), 313-322.

[St] E. N. Stepanova, Extension of continuous functions and metrizability of paracompact p-spaces, Mat. Zametki 53 (1993), 92-101.

[Te] R. Telgársky, On sieve-complete and compact-like spaces, Topology Appl. 16 (1983), 61-68.

[Wh] W. Whitt, Continuity of Markov Processes and Dynamic Programs, Yale University, 1975.

[WW] H. H. Wicke and J. M. Worrell, Jr., On the open continuous images of paracompact Čech-complete spaces, Pacific J. Math. 37 (1971), 265-276.

[Za] S. K. Zaremba, Sur certaines familles de courbes en relation avec la theorie des equations differentielles, Rocznik Polskiego Tow. Matemat. 15 (1936), 83-105.

Academy of Sciences, Institute of Mathematics, Štefánikova 49, 81473 Bratislava, SLOVAKIA

E-mail address: hola@mat.savba.sk

Department of Mathematics and Computer Science, University of North Carolina at Pembroke, Pembroke, NC 28372, USA

E-mail address: laszlo@uncp.edu 